\title{
Breakdown of the Kratky-Porod Wormlike Chain Model for Semi- flexible Polymers in Two Dimensions
}

\author{
Hsiao-Ping Hsu ${ }^{1}$, Wolfgang Paul ${ }^{2}$ and K. Binder ${ }^{1}$ \\ 1 Institut für Physik, Johannes Gutenberg Universität Mainz, \\ Staudinger Weg 7, 55099 Mainz, Germany \\ 2 Theoretische Physik, Martin-Luther-Universität Halle Wittenberg, \\ von Senckendorffplatz 1, 06120 Halle, Germany
}

PACS 82.35.Lr - Physical properties of polymers

PACS 87.15.A- - Theory, modeling, and computer simulation

PACS 36.20.Ey - Conformation (statistics and dynamics)

PACS 82.35.Gh - Polymers on surfaces; adhesion (see also 68.35.Np Adhesion in surfaces and interfaces)

\begin{abstract}
By large-scale Monte Carlo simulations of semiflexible polymers in $d=2$ dimensions the applicability of the Kratky-Porod model is tested. This model is widely used as "standard model" for describing conformations and force versus extension curves of stiff polymers. It is shown that semiflexible polymers in $d=2$ show a crossover from hard rods to self-avoiding walks, the intermediate Gaussian regime (implied by the Kratky-Porod model) is completely absent. Hence the latter can also describe force versus extension curves only if the contour length is only a few times larger than the persistence length. Consequences for experiments on biopolymers at interfaces are briefly discussed.
\end{abstract}

Characterizing the flexibility or stiffness of polymer chains is of basic importance for describing their structure and dynamics, and hence relevant for understand' ing the functions of biopolymers, as well as the application properties of synthetic polymers [1-4]. Moderately 'stiff ("semiflexible") macromolecules behave like rods on ' small scales, and one captures this behavior by the concept of the so-called "persistence length" $\ell_{p}$. For larger length scales, entropic flexibility prevails and random coil' like structures occur. Important examples for such stiff biopolymers are DNA, some proteins, actin, neurofilaments, but also mesoscopic objects such as viruses [5-7]. The experimental study of such biopolymers and the interpretation of these observations by models is a very active topic of research $\{$ e.g. 8 [17 $\}$. In particular, the conformation of these biopolymers can be directly visualized by electron microscopy (EM) or scanning force microscopy (SFM) techniques when such polymers are adsorbed on substrates [8 10, 12, 14, 17]; by atomic force microscopy (AFM) also force versus extension curves can be measured [11,13]. The same methods also work for synthetic polymers such as molecular brushes [18, where stiffness is controlled by the length of side chains [19].
The standard theoretical model, that is almost exclusively used \{e.g. 20 31] $\}$ to interpret these experiments is the simple "wormlike chain (WLC) model" 32,33 . Its Hamiltonian is, in the continuum limit,

$$
\frac{\mathcal{H}}{k_{B} T}=\frac{\kappa}{2} \int_{0}^{L} d t\left(\frac{d^{2} \vec{r}(t)}{d t^{2}}\right)^{2} .
$$

Here the curve $\vec{r}(t)$ describes the linear macromolecule, $t$ is a coordinate along its contour which has the length $L$. We choose units such that $k_{B} T=1$, and the bending stiffness $\kappa$ then is $\kappa=\ell_{p} / 2$, in $d=2$ dimensions. In this paper we shall focus on the case of chains confined to twodimensional geometry, since this case is relevant for the EM and SFM imaging techniques, and also the subject of numerous theoretical studies (e.g. [25, 28, 30]). However, the applicability of eq. (1) in principle is questionable, since it neglects excluded volume between the repeat units of the chain completely. Thus, eq. (11) yields the end-toend distance of the polymer chains as

$$
\left\langle R^{2}\right\rangle=2 \ell_{p} L\left\{1-\frac{1}{n}[1-\exp (-n)]\right\} \quad, \quad n=L / \ell_{p} \quad,
$$


and hence for $n \gg 1$ the chain behaves like a Gaussian coil $\left(\left\langle R^{2}\right\rangle=2 \ell_{p} L\right)$ while for $n<1$ it is essentially a rigid rod of length $L$. The bond-autocorrelation function shows then a simple exponential decay,

$$
g(t)=\left\langle\vec{a}_{i} \cdot \vec{a}_{i+s}\right\rangle=\ell_{b}^{2} \exp \left(-t / \ell_{p}\right) \quad, \quad t=s \ell_{b},
$$

where we now consider a chain where $N_{b}$ bonds of length $\ell_{b}$ connect repeat units at sites $\vec{r}_{i}, \vec{a}_{i}=\vec{r}_{i+1}-\vec{r}_{i},\left|\vec{a}_{i}\right|=\ell_{b}$; so $L=N_{b} \ell_{b}$. Finally, if one considers the effect of a force $f$ acting on one chain end (the other being fixed at the origin), by adding a term - $f X$ to the Hamiltonian ( $X$ being the $x$-component of the end-to-end distance), one obtains from eq. (11) the force vs. distance relation to a very good approximation, in $d=2[29]$

$$
f \ell_{p}=\frac{1}{8}\left[6 \frac{\langle X\rangle}{L}-1+\left(1-\frac{\langle X\rangle}{L}\right)^{-2}\right] .
$$

Since various experimental data have been described by eqs. (2)-(4) with some success adjusting parameters such as $\ell_{p}$ and $L$, it is widely believed that the basic KratkyPorod model, eq. (1), describes semiflexible chains accurately, and a large body of work is concerned with various refinements of this model \{see e.g. [26 30]

However, in the present Letter we show that in fact in $d=2$ the validity of the Kratky-Porod model in the good solvent regime is very restricted, it always holds only up to contour lengths $L$ of a few times $\ell_{p}$, irrespective how large the persistence length $\ell_{p}$ is. In particular, a regime of $L$ where Gaussian statistics holds, $\left\langle R^{2}\right\rangle=2 \ell_{p} L$, in $d=$ 2 is completely absent, unlike the case of $d=3$, where for very large $\ell_{p}$ a double crossover (rods $\rightarrow$ Gaussian coils $\rightarrow$ non-Gaussian swollen coils) is established both experimentally [34] and theoretically [35]. Also eq. (4) breaks down for $L \gg \ell_{p}$, irrespective how large $\ell_{p}$ is. In $d=2$, we will show that

$$
\left\langle R^{2}\right\rangle^{1 / 2} \propto \ell_{p}^{1 / 4} L^{3 / 4} \quad, L>\ell_{p}
$$

and $g(t) \propto t^{-1 / 2}$, for $t>\ell_{p}$, rather than eq. (3). The latter result is consistent with the scaling prediction 36. $g(t) \propto t^{-\beta}$ with $\beta=2(1-\nu)$ where the Flory exponent $\nu=3 / 4$ in $d=2$, as written already in eq. (5).

There has been evidence for the scaling $\left\langle R^{2}\right\rangle^{1 / 2} \propto L^{3 / 4}$ for not so stiff polymers such as single stranded DNA in $d=2$ dimensions, see e.g. [9,10,16, but it has been widely believed that for very stiff polymers excluded volume interactions (that cause the nontrivial exponent $\nu=3 / 4$ rather than the Gaussian result $\nu=1 / 2$ which follows from eq. (2) ) can be neglected, except for extremely long chains. We will show, however, that excluded volume effects set in strongly already for $L \approx 5 \ell_{p}$, invalidating the straightforward use of eqs. (2)-(4) for many cases of interest.

We carried out Monte Carlo simulations of self-avoiding walks (SAWs) on the square lattice, applying an energy $\varepsilon_{b}$ if the orientation of bond vector $\vec{a}_{i}$ differs (by $\pm \pi / 2$ ) from that of $\vec{a}_{i-1}$, and using the pruned-enriched Rosenbluth (a)

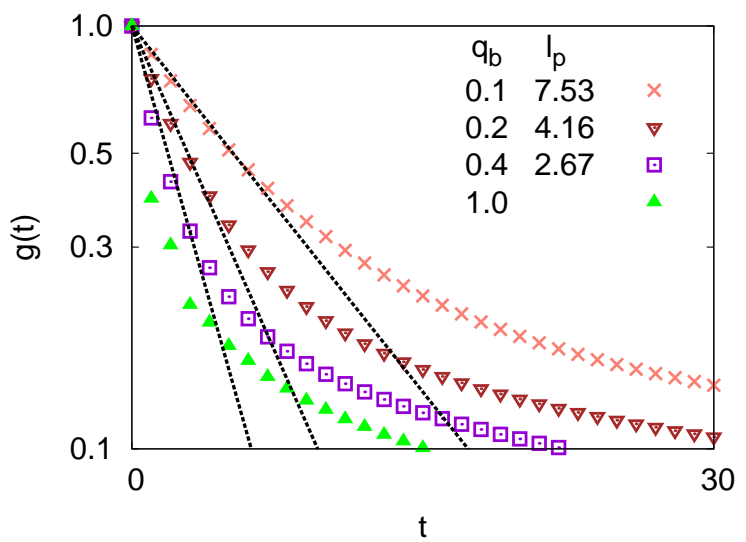

(b)

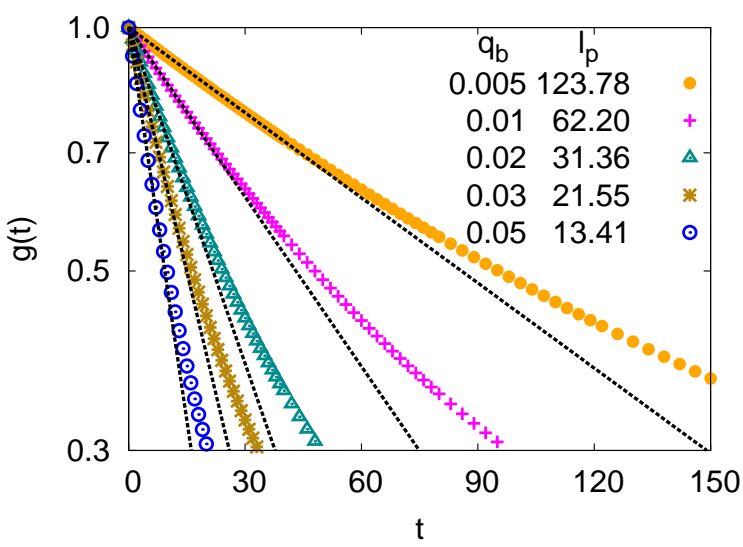

(c)

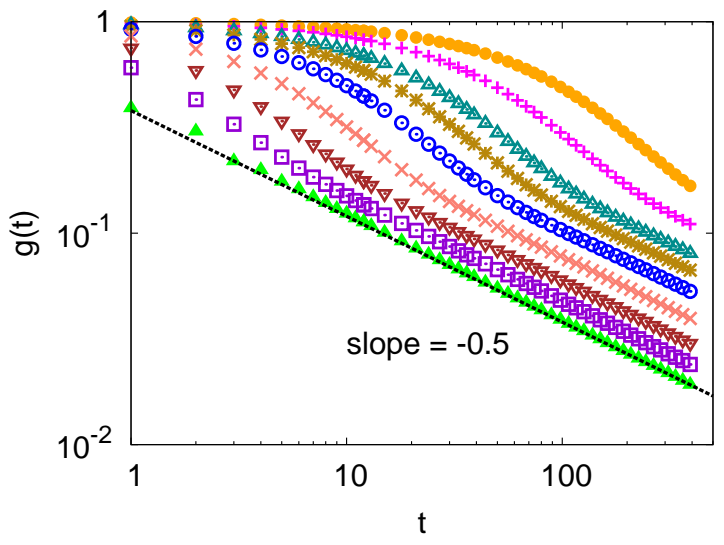

Fig. 1: Semi-log plots of the bond-correlation $g(t)$ vs. the contour length $t$, for the ranges (for a definition of the parameters see eq. (6) $0.1 \leq q_{b} \leq 1.0$ (a) and $0.005 \leq q_{b} \leq 0.05$ (b). The data are taken for $L=25600$ and $b=1$, averaging over the site $i$ in eq. (3). Straight lines indicate fits of the initial decay of $g(t)$ to eq. (3)). The resulting values of $\ell_{p}$ are quoted in the figure. (c) Log-log plot of $g(t)$ vs. $t$, for $q_{b}=0.005$ to 1.0 (from above to below). The straight line shows a fit of the data for $q_{b}=1$ and $t \geq 10$ to the power law $g(t) \propto t^{-0.5}$. 
method [35, 37, 38. The partition function of SAWs with $N_{b}$ steps and $N_{\text {bend }}$ local bends is

$$
Z_{N, N_{\text {bend }}}\left(q_{b}, b\right)=\sum_{\text {config }} C\left(N_{b}, N_{\text {bend }}, X\right) q_{b}^{N_{\text {bend }}} b^{X}
$$

where $q_{b}=\exp \left(-\varepsilon_{b} / k_{B} T\right), b=\exp \left(f / k_{B} T\right)$ and $X$ is the $x$-component of the end-to-end distance (assuming that the force $f$ acts in the $+x$-direction). In experiments where a force is applied to an end of a strongly adsorbed chain, that takes essentially two-dimensional conformations, it is possible to direct this force either perpendicular or parallel to the surface; only the latter case is considered here. Note $q_{b}=1$ for flexible chains (standard SAWs) and $b=1$ in the absence of the force $f$. We generated data for $C\left(N_{b}, N_{\text {bend }}, X\right)$ for $0.005 \leq q_{b} \leq 1.0$ and $N_{b} \leq 25600$.

Fig. 1 shows the bond-orientational correlations (for the case $f=0)$. For rather flexible chains, $q_{b}=0.4$, there are at best a few values $t=1,2,3$ compatible with an exponential decay (we use $\ell_{b}=1$ here and in the following). For small $q_{b}$, eq. (3) has a more extended range of applicability, and $\ell_{p}$ strongly increases when $q_{b}$ decreases, $\ell_{p} \approx 0.61 / q_{b}$. But the asymptotic decay always is the expected power law (fig. 1(c)). As has been emphasized recently [39, in the presence of excluded volume "the" persistence length is a somewhat ill-defined concept; for the present model, $\ell_{p}$ henceforth is defined from the initial slope of the curves $\ln g(t)$ vs. $t$ as $t \rightarrow 0$.

Fig. 2 presents a test of eq. (2). While eq. (2) trivially works for $L<\ell_{p}$ (the rod-like regime), significant deviations become visible for $L>5 \ell_{p}$, irrespective of how large $\ell_{p}$ is, as the scaling plot (fig. 2(b)) shows. In contrast to occasional claims in the literature [12, a regime of Gaussianlike coils is completely absent in $d=2$. This result can be rationalized by the proper adaptation of Flory-type arguments 40 to $d=2$. The free energy of a stiff chain is taken as the sum of an elastic energy $\left(R^{2} / \ell_{p} L\right)$ and the enthalpy due to repulsions, proportional to the 2nd virial coefficient $\left(v_{2}=\ell_{p}^{2}\right.$ [41]; prefactors of order unity are suppressed throughout)

$$
\Delta F=R^{2} /\left(\ell_{p} L\right)+v_{2} R^{2}\left[\left(L / \ell_{p}\right) / R^{2}\right]^{2} .
$$

In $d=2$, the "volume" of a chain of radius $R$ scales like $R^{2}$, and the density of the $n=L / \ell_{p}$ subunits is $n / R^{2}$ in this volume. Minimizing $\Delta F$ with respect to $R$ yields eq. (5). The minimum length $L$ where eq. (5) holds is found when the enthalpic term in eq. (7) is unity for $R^{2}=\ell_{p} L$, i.e. for $L^{*}=\ell_{p}^{3} / v_{2}=\ell_{p}$, and there the rod-like regime starts: this argument shows that we should expect a single crossover from rods to SAWs, as seen in fig. 2(b), unlike the $d=3$ case [35, 40].

How then can we understand the apparent success (suggested in the literature) of the Porod-Kratky model to analyze force-extension curves in $2 \mathrm{~d}$ ? In fig. 3 we show some of our results on force vs. extension curves in $d=2$ and compare our data to the theoretical prediction based on (a)

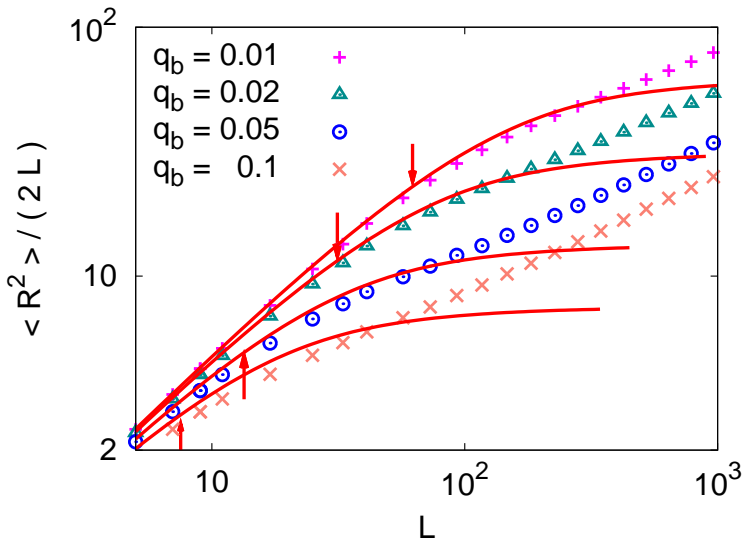

(b)

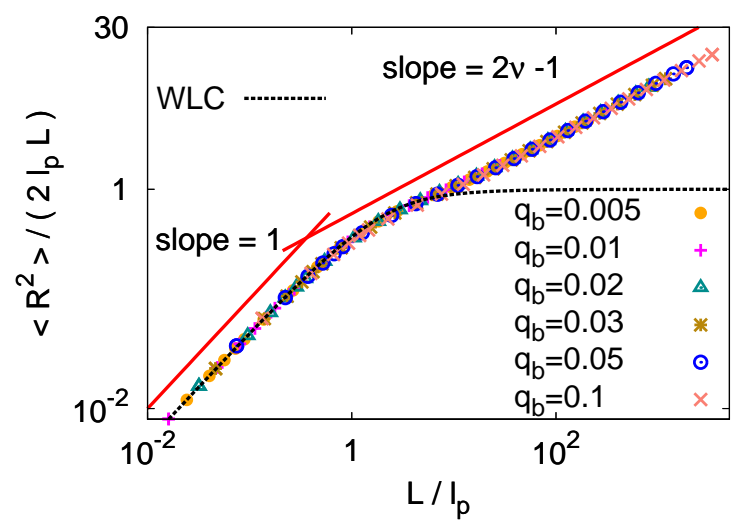

Fig. 2: $\log$-log plot of $\left\langle R^{2}\right\rangle /(2 L)$ versus $L=N_{b} \ell_{b}$ (a) and $\log -\log$ plot of $\left\langle R^{2}\right\rangle /\left(2 \ell_{p} L\right)$ versus $L / \ell_{p}$ (b), for $b=1$ and several choices of $q_{b}$, as indicated. Full curves show the WLC prediction, eq. (2), using $\ell_{p}$ (highlighted by arrows in (a)) from fig. 1(a)(b) as an input. Straight lines in (b) indicate the power laws in the rod regime $\left(\left\langle R^{2}\right\rangle=L^{2}\right)$ and the SAW regime $\{$ eq. (5) $\}$, respectively.

the WLC model, eq. (4). Here the persistence length estimates quoted in Figs. 1(a)(b) were used, so we can compare our simulation results that are based on eq. (6) to the prediction, eq. (4), without adjusting any parameter whatsoever. One can see that the latter equation works only approximately (fig. [3(a)) for very stiff chains in a very restrictive range of contour lengths, where we can deduce from a detailed inspection of the data that $6<L / \ell_{p}<10$ must be fulfilled: if $L / \ell_{p}$ is too small, the chain behaves as a flexible rod, which can be oriented by a force but not stretched; if $L / \ell_{p}$ is too large, excluded volume effects invalidate eq. (4), similarly as eq. (2) fails then. For $q_{b}=0.4$, the chains have hardly any rod-like regime as Fig. 1(a) reveals, $\ell_{p}$ is less than three lattice spacings, and so large deviation from eq. (4) are no surprise, of course. For small $q_{b}$, where for the chosen values of $L=200$ in 
(a)

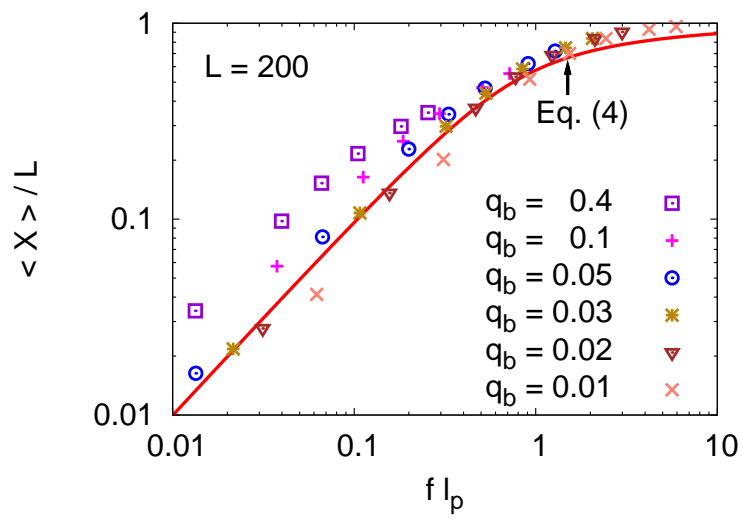

(b)

(c)
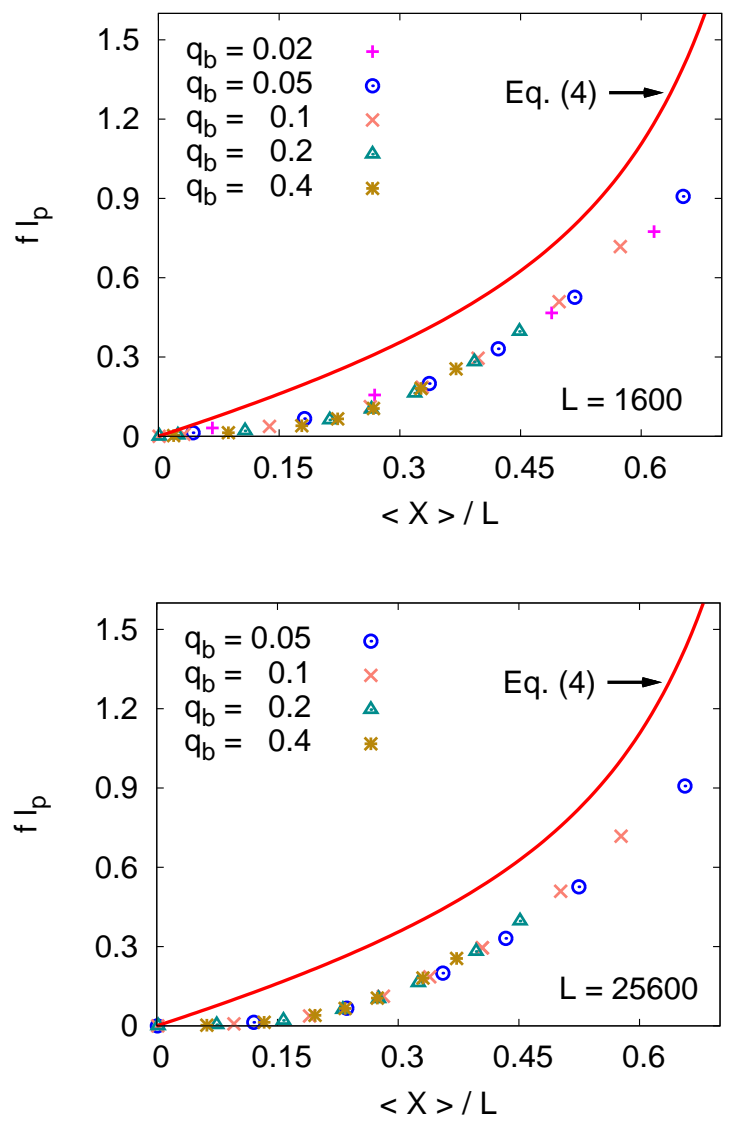

Fig. 3: $\log -\log$ plot of $\langle X\rangle / L$ vs. $f \ell_{p}$ for $L=200$. Eq. (4) is shown by the full curve for comparison (a). Rescaled force $f \ell_{p}$ plotted against $\langle X\rangle / L$ for $L=1600$ (b) and $L=25600$ (c). Various values of $q_{b}$ are shown as indicated.

Fig. 3(a) $L$ is only a few times larger than $\ell_{p}$ (recall $\ell_{p} \simeq 62$ for $q_{b}=0.01$, Fig. 1 (b)), the deviations of the data from eq. (44) go into the opposite direction $\left(\langle X\rangle / L\right.$ for $f \ell_{p}<1$ is smaller than predicted by eq. (4), while $\langle X\rangle / L$ is larger than predicted if $\ell_{p}$ is small). This finding implies that for $L=200$ and intermediate values of $\ell_{p}$, the observed variation of $\langle X\rangle / L$ with $f \ell_{p}$ is close to the predicted one, for the intermediate range of $L / \ell_{p}$ quoted above, but this agreement is somewhat accidental.

Note also that a sensible test of the Kratky-Porod model (which is a continuum model) by our discrete lattice model is only possible for forces such that $f \ell_{p}<1$, since important deviations between discrete chain models and the Kratky-Porod model occur 26] when the so-called deflection length $\lambda \propto\left(f \ell_{p}\right)^{-1 / 2}$ of worm-like chains becomes smaller than the bond length $\ell_{b}$. Thus our data do not converge to eq. (4) even for large $f \ell_{p}$, although for very strongly stretched chains $(\langle X\rangle / L)$ close to unity) excluded volume effects must become irrelevant.

If $L$ is very large, such a crossing of the simulated curves for $\langle X\rangle / L$ as function of $f \ell_{p}$ with eq. (4) when $\ell_{p}$ is varied does no longer occur (Fig. 3 (b)(c)). The simulation results for $\langle X\rangle / L$ are now always significantly larger than the prediction, eq. (4), particularly for small values of $f \ell_{p}$. This huge discrepancy for small values of $f \ell_{p}$ can be understood readily in terms of a linear response argument: actually, eq. (44) is found from adding a term $-f X$ to the Hamiltonian, eq. (11). Therefore it is straightforward to derive, in the limit $f \rightarrow 0$, the linear response relation

$$
\partial\langle X\rangle /\left.\partial f\right|_{f=0}=\left\langle X^{2}\right\rangle_{f=0} .
$$

Since $\left\langle X^{2}\right\rangle_{f=0}=\left\langle R^{2}\right\rangle / 2$, where according to the KratkyPorod model \{eq. (2) $\}$ for $L \gg \ell_{p}$ we have simply $\left\langle R^{2}\right\rangle=$ $2 \ell_{p} L$, we conclude that $\langle X\rangle=\left\langle X^{2}\right\rangle f=\ell_{p} f L$ (in agreement with the Taylor expansion of eq. (4) to first order in $\langle X\rangle / L$, as it must be, of course). However, in $d=2$ for vanishing force and $L \gg \ell_{p}$ the relation $\left\langle X^{2}\right\rangle=\ell_{p} L$ must be replaced by $\left\langle X^{2}\right\rangle \propto \ell_{p}^{1 / 2} L^{3 / 2}$, as is readily seen from eq. (5). Therefore we predict for the linear response regime a very different scaling for the force-extension behavior, namely

$$
\langle X\rangle / L \propto \ell_{p}^{1 / 2} L^{1 / 2} f .
$$

This relation is tested in Fig. 4. A wide range of choices of contour lengths $L=N_{b} \ell_{b}$ and several choices of $q_{b}$ and hence $\ell_{p}$ (the relation between $q_{b}$ and $\ell_{p}$ is quoted in Fig. 1(a)(b)) are included. An interesting issue also is the regime of relative extensions over which linear response holds: while eq. (4) implies a linear response regime applying almost up to $\langle X\rangle / L \approx 0.3$, irrespective of $\ell_{p}$, we suggest that the linear response breaks down if $\langle X\rangle^{2} \approx\left\langle X^{2}\right\rangle$, i.e. for $\langle X\rangle / L \propto\left(\ell_{p} / L\right)^{1 / 4} \rightarrow 0$ as $\ell_{p} / L \rightarrow 0$. In the nonlinear regime, fig. [3(b)(c) suggests that $\langle X\rangle / L$ can be described by some universal function of $\ell_{p} f$, that does not depend on $\ell_{p}$ : this is the universality of $d=2$ SAWS, not the Kratky-Porod model.

Of course, the scaling $\langle X\rangle \propto L^{3 / 2} f$ for small $f$ is consistent with the scaling behavior proposed by Pincus 42 . for stretched flexible polymers in the presence of excluded volume

$$
\langle X\rangle=R_{0} F\left(R_{0} / \xi_{p}\right)
$$




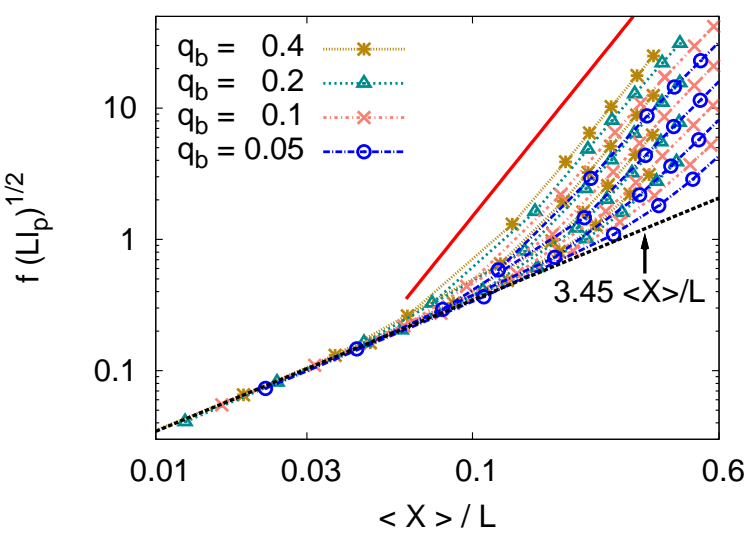

Fig. 4: Log-log plot of $f\left(L \ell_{p}\right)^{1 / 2}$ vs. $\langle X\rangle / L$, including several values of $q_{b}$ as indicated, and data for $N_{b}=400,1600,6400$, and 25600 (from bottom to top at the right side of the diagram, respectively). Straight dotted line (black) indicate the linear response behavior, and straight solid line (red) indicate the non-linear behavior, i.e. $f \propto\langle X\rangle^{3}$ (see text).

where $R_{0}$ is the radius of chain in the absence of a stretching force, $F\left(R_{0} / \xi_{p}\right)$ is a scaling function, and $\xi_{p} \propto 1 / f$ is the size of "Pincus blobs", and hence in the linear response regime $\langle X\rangle \propto R_{0}^{2} f$, i.e. eq. (9) results. The condition that $\langle X\rangle / L$ is of order unity then leads to 42 $\langle X\rangle \propto f^{1 / \nu-1}=f^{1 / 3}$ in $d=2$ dimensions, i.e, a strongly non-linear relation between $f$ and $\langle X\rangle$. This power law in

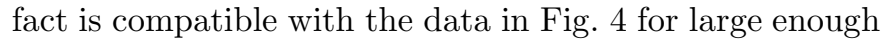
$\langle X\rangle / L$.

In conclusion, we have shown that in $d=2$ dimensions the Kratky-Porod model, that is ubiquitously used to analyze the internal end-to-end distances of biopolymers such as DNA \{e.g. 15, 17] $\}$ or of synthetic polymers such as the bottle brushes $\{$ e.g. [18] $\}$ and to analyze force versus extension curves $\{$ e.g. [11, 13] $\}$ has a very limited validity: it trivially describes the rod-like regime, $L \leq \ell_{p}$, but this regime is not useful in the context of such measurements, which are devoted to understanding the dependence of the persistence length on various parameters (such as particular amino acid sequences in DNA, or side chain length in bottle brushes, etc.). In $d=2$, a regime where Gaussian statistics (requiring $L \gg \ell_{p}$ ) holds is completely absent.

Our findings imply that conformations of semiflexible polymers in $d=2$ (equilibrated surface adsorbed case) depend on their relative length $L / \ell_{p}$ very differently from the case $d=3$ (dilute bulk solution). Thus there is no direct way to infer properties ( $\mathrm{such}$ as $\ell_{p}$ ) in the bulk from measurements on surface adsorbed chains: there is no simple relation between the effective persistence lengths either \{in our model $\ell_{p} \propto 1 /\left(4 q_{b}\right)$ for $d=3$ but $\ell_{p} \cong 0.61 / q_{b}$ in $d=2$ for $\left.q_{b} \rightarrow 0\right\}$.

Going beyond the strictly 2-dimensional case, exploring the crossover to weak adsorption (chains with dangling non-adsorbed "tails" and "loops" in addition to adsorbed "trains") will be intriguing. Also, the effects of excluded volume on force versus extension curves when strongly adsorbed chains are pulled off a surface in the direction normal to the surface by an AFM tip need to be studied carefully. Thus, much further work is needed for a better modeling of biopolymers and other stiff polymers at interfaces, and on the interpretation of the corresponding experiments.

The effects studied in our work should also be relevant when one studies semiflexible chains confined to the surface of a sphere or its interior [43, a problem believed to be of great biological relevance.

Acknowledgements: We thank the Deutsche Forschungsgemeischaft(DFG) for support (grant No SFB625/A3) and the Jülich Supercomputing Centre (JSC) for computer time at the NIC Juropa supercomputer.

\section{REFERENCES}

[1] GROSBERG A. Yu. and KHOKHLOV A. R., Statistical Physics of Macromolecules (AIP Press, Woodbury) 2002.

[2] WITten T. A., Rev. Mod. Phys., 70 (1988) 1531.

[3] RUbinstein M. and COLBY R. H., Polymer Physics (Oxford University Press, Oxford) 2003.

[4] ZWOlAK M. and DI Ventra M., Rev. Mod. Phys., 80 (2008) 141.

[5] BUStamante C., MARKO J. F., SigGia E. D. and SMITH S., Science, 265 (1994) 1599.

[6] KÄS J., STREY H., TANG J. X., FINGER D., EZZELL R., SACKMANN E. and JANMEY P. A., Biophys. J., 70 (1996) 609.

[7] OBER C. K., Science, 288 (2000) 448.

[8] STOKKE B. T. and BRANT D. A., Biopolymers, 30 (1990) 1161.

[9] MAIER B. and RÄDLER J. O., Phys. Rev. Lett., 82 (1999) 1911.

[10] MAIER B. and RÄDLER J. O., Macromolecules, 33 (2000) 7185.

[11] DESSINGES M.-N., MAIER B., ZHANG Y., PELITI M., Bensimon D. and CROQUetTe V., Phys. Rev. Lett., 89 (2002) 248102.

[12] YOSHINAGA N., YOSHIKAWA K. and KIDOAKI S., J. Chem. Phys., 116 (2002) 9926.

[13] SEOL Y., SKInNeR G. M. and VISSCHER K., Phys. Rev. Lett., 93 (2004) 118102.

[14] VAlle F., FAVRE M., DE LOS RIOS P., ROSA A. and DIETLER G., Phys. Rev. Lett., 95 (2005) 158105.

[15] MOUKHTAR J., FONTAINE E., FAIVREMOSKalenko C. and ARneOdo A., Phys. Rev. Lett., 98 (2007) 178101.

[16] RECHENDORFF K., WITZ G. ADAMCIK J. and DIETLER G., J. Chem. Phys., 131 (2009) 095103.

[17] MOUKHTAR J., FAIVRE-MOSKALENKO C., MILANI P., AUDIT B., VAILLANT C., FONTAINE E., MONGELARD F., LAVOREL G. ST-JEAN P., BOUVET P., ARGOUL F. and ARNEODO A., J. Phys. Chem. B, 114 (2010) 5125. 
[18] GUNARI N., SCHMIDT M. and JANSHOFF A., Macromolecules, 39 (2006) 2219.

[19] SHEIKO, S. S., SUMERLIN, B. S., MATYJASZEWSKI, K., Prog. Polym. Sci., 33 (2008) 759.

[20] WINKLER R. G., REINEKER P. and HARNAU L., J. Chem. Phys., 101 (1994) 8119.

[21] MARKO J. F. and SIGGIA E.D., Macromolecules, 28 (1995) 8759.

[22] KROY K. and FREY E., Phys. Rev. Lett., 77 (1996) 306.

[23] HA B.-Y. and ThiRumAlAi D., J. Chem. Phys., 106 (1997) 4243.

[24] RivetTi C., WALKER C. and BUStAMANTE C., J. Mol. Biol., 280 (1998) 41.

[25] LAMURA A., BURKHARDT T. W. and GOMPPER G., Phys. Rev. E, 64 (2001) 061801.

[26] LIVADARU L., NETZ R. R. and KREUZER H., Macromolecules, 36 (2003) 3732.

[27] WinkleR R. G., J. Chem. Phys., 118 (2003) 2919.

[28] STEPANOW S., Eur. Phys. J. B, 39 (2004) 499.

[29] PRASAD A., HORI Y. and KONDEV J., Phys. Rev. E, 72 (2005) 041918.

[30] MOUKhtAR J., VAILlant C., AUdiT B. and ARNEODO A., EPL, 86 (2009) 48001.

[31] TOAN N. M. and THIRUMALAI D., Macromolecules, 43 (2010) 4394.

[32] KRATKY O. and POROD G., J. Colloid Sci., 4 (1949) 35.

[33] SAItO N., TAKAHASHI K., YUNOKI Y., J. Phys. Soc. Jpn., 22 (1967) 219.

[34] NORISUYE T. and FUJITA H., Polymer. J., 14 (1982) 143.

[35] HSU H.-P., PAUL W. and BINDER K., EPL, 92 (2010) 28003.

[36] SCHÄFER L., OSTENDORF A. and HAGER J., J. Phys. A: Math. Gen., 32 (1999) 7875.

[37] GRASSBERGER P., Phys. Rev. E, 56 (1997) 3682.

[38] BAStOlla U. and GRASSBERGER P., J. Stat. Phys., 89 (1997) 1061.

[39] HSU H.-P., PAUL W. and BINDER K., Macromolecules, 43 (2010) 3094.

[40] NETZ R. R. and ANDELMAN D., Phys. Rep., 380 (2003) 1.

[41] A rod of $\ell_{p}$ subsequently occupied lattice sites on the square lattice blocks a square size $\ell_{p}^{2}$ for occupation to another rod, oriented perpendicularly to the first one.

[42] PINCUS P., Macromolecules, 9 (1976) 386.

[43] MORRISON G. and THIRUMALAI D., Phys. Rev. E, 79 (2009) 011924. 